\title{
A REINTERPRETATION OF PHASE VELOCITY DATA BASED ON THE GNOME TRAVEL TIME CURVES
}

\author{
By Stewart W. Smith
}

\begin{abstract}
Significant lateral variations in upper mantle velocities across the western U. S. were observed in the GNOME experiment. This makes necessary a reinterpretation of crustal thickness measurements made with the assumption that velocities in the various layers of the crust remain constant while their thickness changes. Four examples of the work of Ewing and Press have been reinterpreted. The crust is thinner $(30 \mathrm{~km})$ in the Basin and Range Province and thicker $(55 \mathrm{~km})$ under the Rocky Mountains than indicated by previous interpretations.
\end{abstract}

\section{INTRODUCTION}

The seismic travel time curves provided by the GNOME experiment show very strong lateral variations in the apparent velocity of the upper mantle. Velocities in southeast Nevada, Arizona and New Mexico appear to be low $(7.7-7.9 \mathrm{~km} / \mathrm{sec})$ while those in Oklahoma, Arkansas and Tennessee are high $(8.2-8.4 \mathrm{~km} / \mathrm{sec})$. A similar effect has been observed in the phase velocity of Rayleigh waves across these regions. At a period of 20 seconds, this velocity is controlled by the thickness of the crust. The lower phase velocities in the west are attributed to a thicker crust.

Ewing and Press (1959) measured the phase velocity of Rayleigh waves across the United States and based their interpretation on a standard crustal section in which the velocities were constant. Observed phase velocities could then be accounted for by varying only the total thickness of this crustal section. Press (1960) applied this method to the California-Nevada region and pointed out the manner in which gravity and refraction data can be used to reduce the ambiguities in the interpretation of phase velocity data. Upper mantle velocities determined from the GNOME experiment will be used as the basis for reinterpretation of four examples of the work of Ewing and Press (1959). A more extensive reinterpretation of this particular set of data has not been attempted because of the narrow period range covered (18-30 seconds). The network of stations set up to record GNOME includes long period instruments at most locations and thus should provide excellent data for a detailed study of the variation in phase velocity across the U.S. by making use of distant earthquake signals.

\section{Dispersion Data}

Phase velocities across the network consisting of Tucson, Boulder and Lubbock are shown in figure 1. The GNOME explosion recorded on the U.S.G.S. profile north from Carlsbad provides a good velocity depth model through the center of this network including a measured shear velocity for the mantle of $4.5 \mathrm{~km} / \mathrm{sec}$, which corresponds to a Poisson's ratio of 0.28 . The theoretical phase velocity curve for the refraction model departs significantly from the observed data. The two will be brought into correspondence if the depth to the $M$-discontinuity is increased by 10 $\mathrm{km}$ (shown by the dashed line in figure 1) or if a low velocity layer is inserted near the base of the crust. Our interpretation using velocities from the GNOME experi- 
ment makes the crust about $55-60 \mathrm{~km}$ thick as compared with the previous value of $47 \mathrm{~km}$ for this region. This is an average thickness over a very complex region, most of which is in the Rocky Mountains.

In Arizona and southern Nevada, travel times from the GNOME experiment interpreted by Herrin (personal communication, 1962) give low $(7.6-7.8 \mathrm{~km} / \mathrm{sec}$ ) apparent velocities for the upper mantle. In this same region, the crustal refraction work of Tatel and Tuve interpreted by Woollard and reported by Steinhart and Meyer (1961) shows an upper mantle velocity of $8.2 \mathrm{~km} / \mathrm{sec}$. There are several ways that this apparent inconsistency in velocity can be resolved, disregarding the possibility of misinterpretation in either case. If we assume that the short range refraction arrivals observed by Tatel and Tuve were from a thin layer of $8.2 \mathrm{~km} / \mathrm{sec}$ material overlying a thicker section of $7.7 \mathrm{~km} / \mathrm{sec}$ material, then one should see a marked dependence of velocity on frequency. Since the refracted arrivals measured in the GNOME experiment were observed at much greater distances and on lower
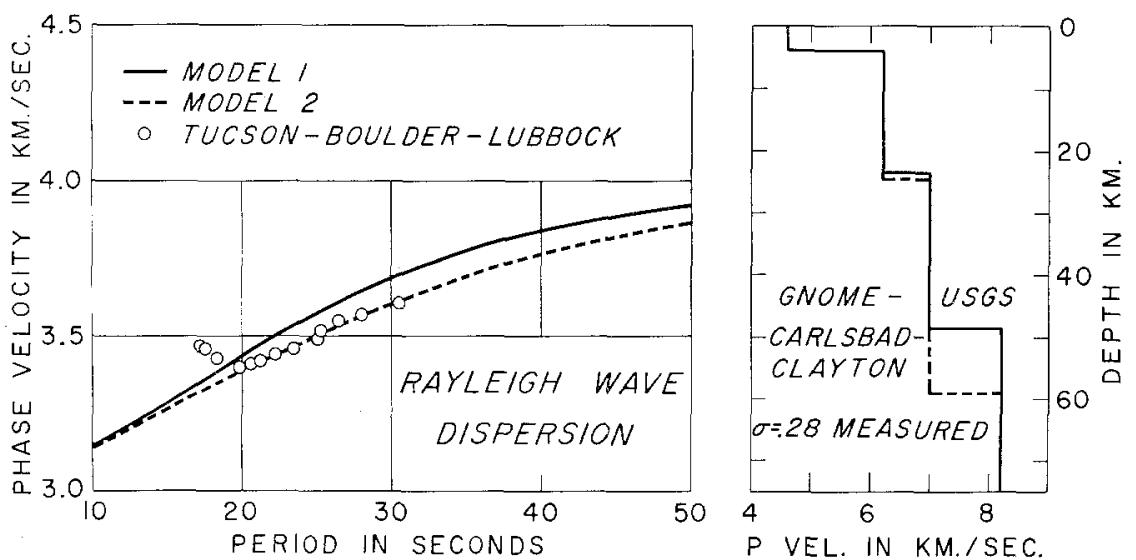

FIG. 1. Experimental phase velocities for the Rocky Mountain region (circles) compared with theoretical phase velocities for the two velocity depth models shown on the right.

frequency instruments, one would expect these longer waves to be unaffected by a thin high velocity layer at the M-discontinuity and show the lower velocity of the underlying layer. Dispersion data for the network of Barrett, Boulder City, and Tucson fits the theoretical phase velocity curve based on the velocity depth model of Woollard moderately well (figure 2) except for periods longer than 25 seconds. If a low velocity layer is inserted directly beneath a thin layer of $8.2 \mathrm{~km} / \mathrm{sec}$ at the base of the crust, (figure 2 dashed line) the fit is even better. If we can accept this hypothesis, that the layer of high velocity material at the M-discontinuity can become very thin, there is no reason to believe that it cannot disappear completely in some regions. It is possible that the so-called intermediate layer $(7.7 \mathrm{~km} / \mathrm{sec})$ observed in California and parts of Nevada should be associated with the upper mantle rather than the crust.

In Nevada, a velocity-depth model based on the U.S.G.S. profile between Fallon and Eureka gave theoretical phase velocities that differ significantly from those observed for the network of Reno, Boulder City, and Eureka (figure 3). If the thickness of the crust is increased by $7 \mathrm{~km}$, the theoretical and observed phase velocity 
curves are brought into agreement (dashed line, figure 3). Since the phase velocity for this network gives an average thickness over almost the entire state of Nevada, this agreement with a single profile in northern Nevada is considered satisfactory. Note that the upper mantle velocity here is only $7.65 \mathrm{~km} / \mathrm{sec}$. If the M-discontinuity

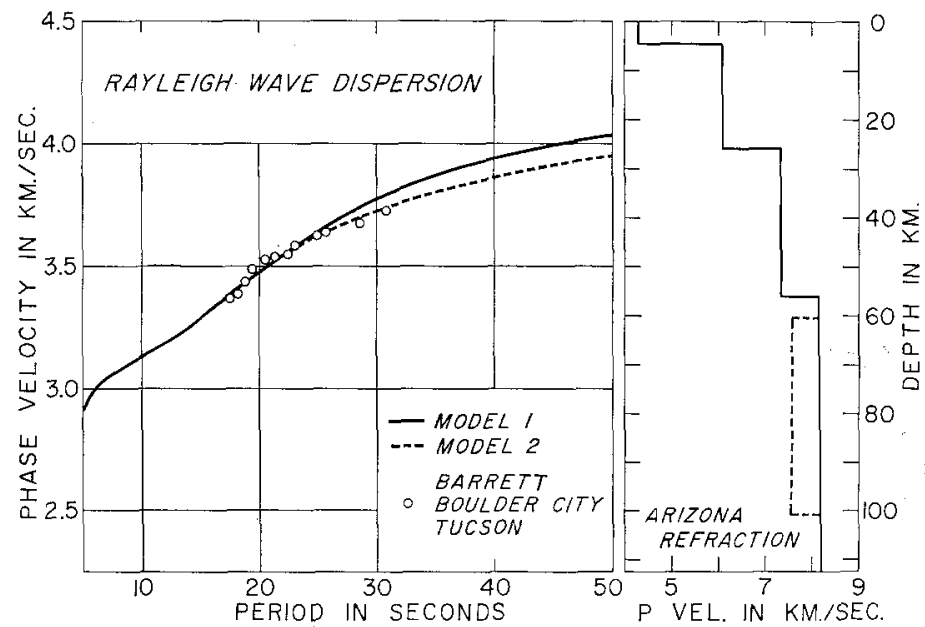

FIG. 2. Experimental phase velocities for the Arizona region (circles) compared with theoretical phase velocities for the two velocity depth models shown on the right.
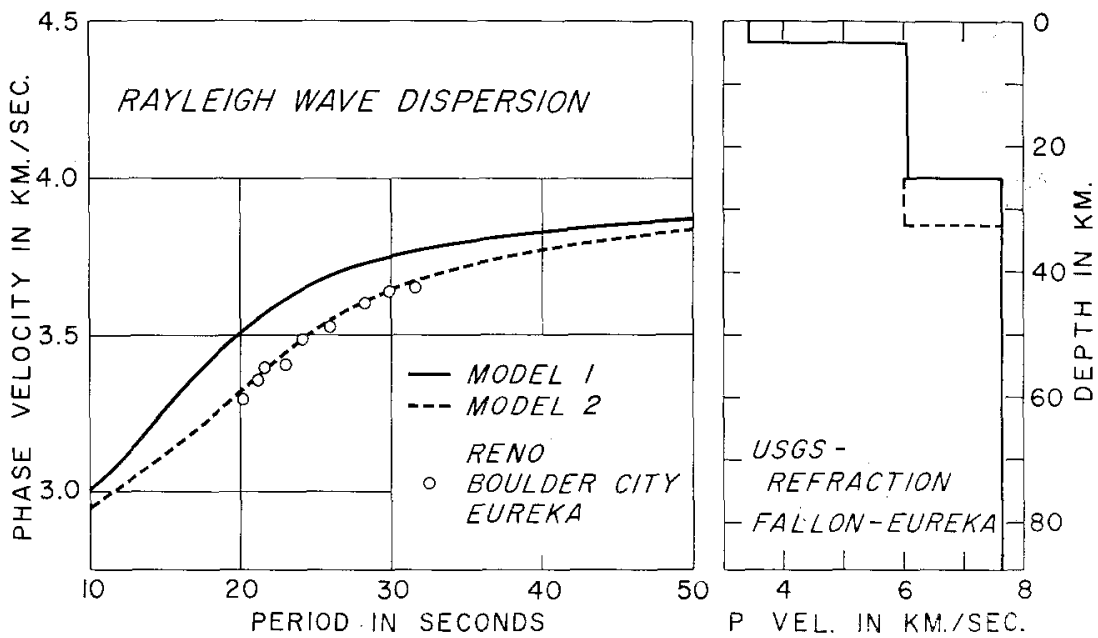

FIG. 3. Experimental phase velocities for the Nevada region (circles) compared with theoretical phase velocities for the two velocity depth models shown on the right.

were a thin high velocity layer in Arizona as mentioned above, it may disappear completely in Nevada. It is possible that the refraction profile between Fallon and Eureka was not long enough to see the M-discontinuity arrival. We attempted to test this hypothesis by putting in a half space of $8.2 \mathrm{~km} / \mathrm{sec}$ material at various depths between 40 and 80 kilometers. The variation in phase velocity for the short range of periods studied was not great, but it appeared that none of the models 
fitted as well as the one given in figure 3 . If a layer of $8.2 \mathrm{~km} / \mathrm{sec}$ exists in this region, it must be deeper than 80 kilometers.

In the region to the east, covered by the network Boulder City, Eureka, Salt Lake City, we found the velocity depth model from the Fallon Eureka profile gave theoretical dispersion curves that fitted the observed data remarkably well, if Poisson's ratio was increased to 0.29 (figure 4 dashed line). The slope of the observed phase velocities is such that no reasonable variation in layer thickness will give a good fit unless the shear velocity is also lowered. It is of interest to note that this value of Poisson's ratio for the western side of the Rocky Mountains is in agreement with that measured north into the Rockies from GNomE.
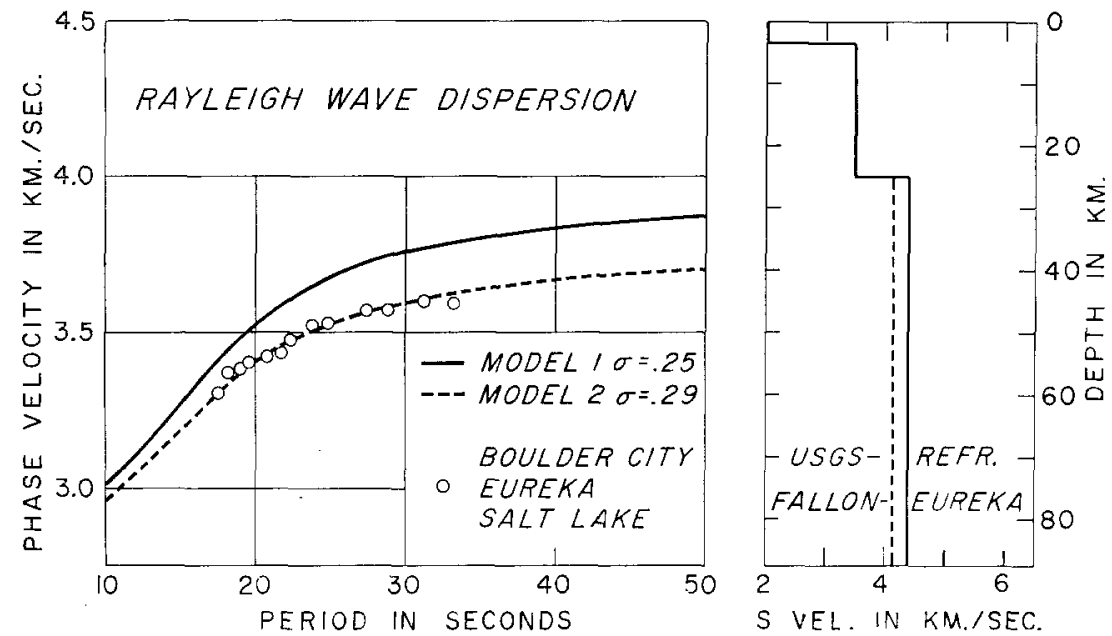

Frg. 4. Experimental phase velocities for the Nevada-Utah region (circles) compared with theoretical phase velocities for the two velocity depth models shown on the right.

\section{Conclusions}

Lateral variations in the velocity of the upper mantle revealed by the GNOME experiment necessitate a reinterpretation of crustal thickness calculations based on the assumption of constant velocity and changing thickness. In four regions studied, the dispersion results could be made consistent with long and short range refraction data. Under the Rocky Mountains the crust is thicker, and the shear velocity lower than was previously thought to be the case. In the Basin and Range province the crust is thinner and the velocity lower with a marked decrease in shear velocity toward the Rocky Mountains. In Arizona a model of a thin high velocity $(8.2 \mathrm{~km} / \mathrm{sec})$ layer at the M-discontinuity immediately overlying a layer of $7.6 \mathrm{~km} / \mathrm{sec}$ gave consistent results for both refraction and dispersion data.

\section{ACKNOWLEDGMENTS}

We are grateful to John H. Healy of the U. S. Geological Survey (Crustal Studies Branch) for making available refraction data for the Nevada and New Mexico regions in advance of publication.

This research was supported by contract AF-49 (638) 910 of the Air Force Office of Scientific Research as part of the Advanced Research Projects Agency project veLA unIForm. 


\section{REFERENCES}

Ewing, M., and F. Press

1959. "Determination of Crustal Structure from Phase Velocity of Rayleigh Waves, Part III: The United States," Bull. Geol. Soc. Am., 70: 229-244.

Press, F.

1960. "Crustal Structure in the California-Nevada Region," J. Geophys. Res., Vol. 65, No. 3, 1039-1051.

Steinhart, J., and R. Meyer

1961. "Explosion Studies of Continental Structure," Carnegie Inst. of Washington, Publication No.62\%.

Caltfornia Institute of Technology

Division of the Geological Sciences

Seismologtcal Laboratory

Pasadena, California

Manuscript received June 30, 1962. 